\title{
Wake Behavior behind Turbine Cascades in Compressible Two-Dimensional Flows
}

\author{
Rainer Kurz \\ Solar Turbines Incorporated, 9330 Sky Park Court San Diego, CA 92123-5398, USA \\ Email: kurz_rainer_x@solarturbines.com \\ Received 1 February 2004
}

\begin{abstract}
The goal of the paper is to describe wake parameters of wakes from turbine cascades in compressible flows especially in planes where the leading edge of the following blade row would be located. Data from experiments with turbine cascades in compressible flow will be used to derive a theoretical approach which describes the wake growth and the recovery of the velocity deficit. The theory is based on similarity assumptions. The derived equations depend on simple and readily available parameters such as overall losses, exit angle, and Mach or Laval number. In compressible turbine flows, the influence of the inviscid flow field is of great importance. In this paper, an approach to take this influence into account when determining the behavior of the wake is presented. Correlations for basic characteristics of wakes in compressible flows are not readily available. Such correlations are necessary as input to unsteady flow and heat transfer calculation procedures for turbomachine blades. Based on available data on wake behavior in the compressible flow behind turbine blades, the correlations presented describe the wake behavior from the trailing edge to the confluence of the wakes of adjacent blades.
\end{abstract}

Keywords and phrases: wake, turbine blades, cascades, transonic flow, compressible flow.

\section{INTRODUCTION}

While CFD codes for turbomachinery flows have become widely available, boundary-layer flows in compressible environments are still a subject of research. Sieber [1], for example, reports significant differences between his very accurate, nonintrusive wake measurements and numerical calculations.

Therefore, it was felt that correlations for basic characteristics of wakes in compressible flows are not readily available. Such correlations are necessary as input for unsteady flow and heat transfer calculation procedures for turbomachine blades.

A review of available literature revealed that numerous papers on wakes in incompressible flows exist. However, the available information on wakes in compressible flows behind turbine blades is very limited. Nevertheless, several sources for experimental data exist (Mee et al. [2], Kurz [3, 4, 5]). Because of the application for turbomachinery, only turbulent wakes are taken into consideration. The presented approach is valid from the trailing edge until the width of the wake causes adjacent wakes to interact.

This is an open access article distributed under the Creative Commons Attribution License, which permits unrestricted use, distribution, and reproduction in any medium, provided the original work is properly cited.

\section{CORRELATION BETWEEN BOUNDARY LAYER DIMENSIONS AND WAKE DIMENSIONS}

In this study, the terminology and principles of the boundary-layer approach for airfoils will be used: the coordinate systems to describe the problem are outlined in Figure 1. The wake will be described in terms of displacement thickness $\delta_{1}$, momentum thickness $\delta_{2}$, and form factor $H_{12}=\delta_{1} / \delta_{2}$.

Although the similarity solutions for the wake cannot be applied, we still can take advantage of the fact that the shape of the wake can be approximated by a power-law approach (Figure 2). We use the function given by Schlichting [6]:

$$
\begin{gathered}
\frac{u(s, r)}{U}=1-k_{1}(s) \cdot f(\eta), \quad f(\eta)=\left(1-\eta^{3 / 2}\right)^{2}, \\
\eta=\frac{r}{k_{2}},
\end{gathered}
$$

yielding the following integrals:

$$
\begin{gathered}
F=\int_{\eta=0}^{1} f(\eta) d \eta=0.45, \\
F_{2}=\int_{\eta=0}^{1} f^{2}(\eta) d \eta=0.3156, \\
F_{3}=\int_{\eta=0}^{1} f^{3}(\eta) d \eta=0.251 .
\end{gathered}
$$




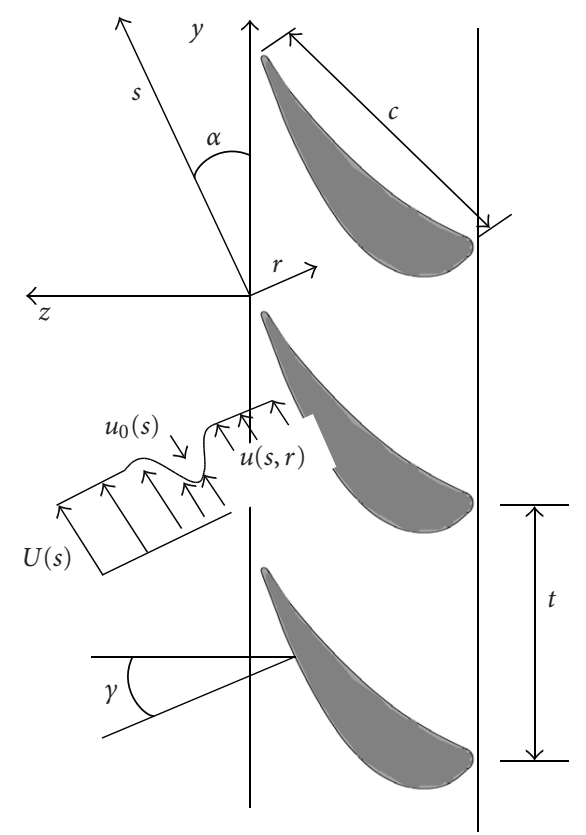

Figure 1: Problem definition and coordinates.

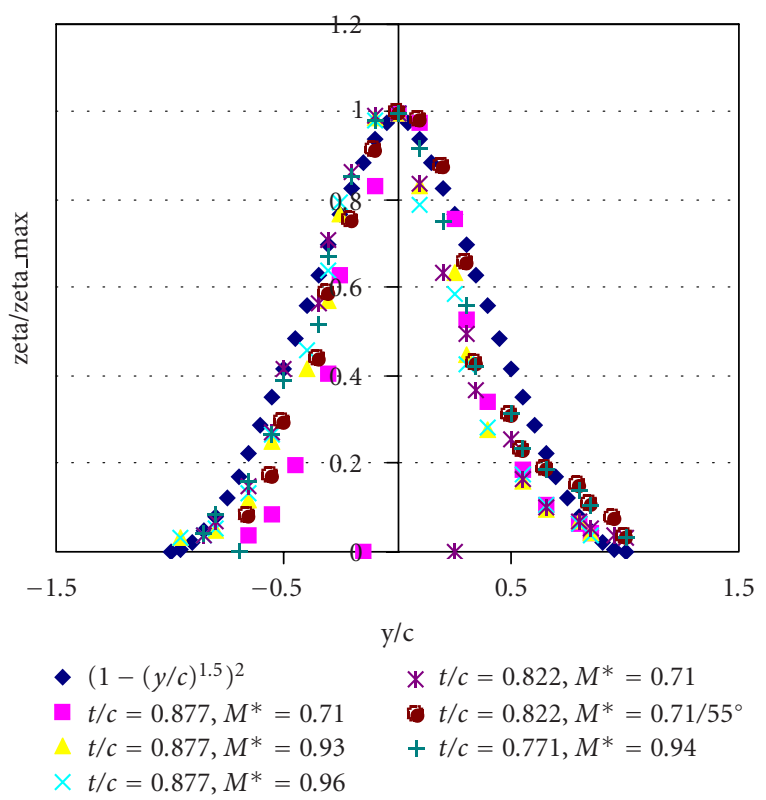

FIGURE 2: Shape of the wake behind a turbine blade, for Laval numbers between 0.71 and 0.96 and pitch-to-chord ratios between 0.771 and 0.877, using identical airfoils (test data from Kurz [5]).

Different to classical approaches, it is not assumed that the parameters $k_{1}$ and $k_{2}$ behave proportionally to $s^{-m}$ and $s^{m}$, respectively, with $m$ being a constant.

With the aforesaid, we are able to calculate the values for $\delta_{1}, \delta_{2}$, and thus $H_{12}$ for a cross, section ( $s=$ const.) of the wake. The integration of $u(r, s)$ for a constant $s$ from $\eta=-1$ to +1 yields

$$
\begin{gathered}
\delta_{1}=k_{1} \cdot k_{2} \cdot 2 \cdot F, \\
\delta_{2}=\delta_{1}-k_{1}^{2} \cdot k_{2} \cdot 2 \cdot F_{2}, \\
H_{12}=\frac{1}{1-0.7013 \cdot k_{1}},
\end{gathered}
$$

which allows us to calculate $k_{1}$ and $k_{2}$ as

$$
\begin{aligned}
k_{1} & =1.426 \cdot\left(1-\frac{1}{H_{12}}\right), \\
k_{2} & =\frac{\delta_{1}}{1.283 \cdot\left(1-1 / H_{12}\right)} .
\end{aligned}
$$

For $\delta_{1}=$ const., the equations for $k_{1}$ and $k_{2}$ satisfy automatically Reichardt's [7] finding that for wakes far behind cylinders $u_{0} * b$ becomes a constant.

For compressible flow, we use the relation

$$
\rho(u)=\frac{p}{R\left(T_{t}-u^{2} / 2 c_{p}\right)}
$$

with $p$ and $T_{t}$ being constant.

We assume that $\rho u$ can be described by a function

$$
\begin{aligned}
& \frac{(\rho u)(s, r)}{(\rho U)_{s}}=1-k_{3}(s) \cdot f(\eta)-k_{1} \cdot f(\eta)+k_{3} \cdot k_{1}^{*} f^{2}(\eta), \\
& k_{3}=1-\frac{\rho_{0}}{\rho_{s}}=1-\frac{\left(1-((\kappa-1) /(\kappa+1)) M_{s}^{* 2}\right)}{\left(1-((\kappa-1) /(\kappa+1))\left(M_{s}^{*}\left(1-k_{1}\right)\right)^{2}\right)} .
\end{aligned}
$$

In this function, the wake width $k_{2}$ will be identical to the wake width in the respective incompressible equation. This leads to the compressible values for $\delta_{1 c}$ with

$$
\delta_{1 c}=\int\left(1-\frac{\rho u}{(\rho U)_{s}}\right) d r=2 k_{2}\left(\left(k_{3}+k_{1}\right) \cdot F-k_{1} k_{3} \cdot F_{2}\right)
$$

and $\delta_{2 c}$ becomes

$$
\begin{aligned}
\delta_{2 c} & =\int \frac{\rho u}{(\rho U)_{s}}\left(1-\frac{u}{U}\right) d r \\
& =2 k_{2}\left(k_{1} F-\left(k_{1} k_{3}+k_{1}^{2}\right) F_{2}+k_{3} k_{1}^{2} F_{3}\right) .
\end{aligned}
$$

Thus, the compressible form factor is

$$
H_{12 c}=\frac{\left(k_{3}+k_{1}\right) \cdot F-k_{1} k_{3} F_{2}}{k_{1} F-\left(k_{1} k_{3}+k_{1}^{2}\right) F_{2}+k_{3} k_{1}^{2} F_{3}} .
$$

This equation for the form factor $H_{12 c}$ is identical with the incompressible form factor for $k_{3}=0$, that is, for incompressible flow. 


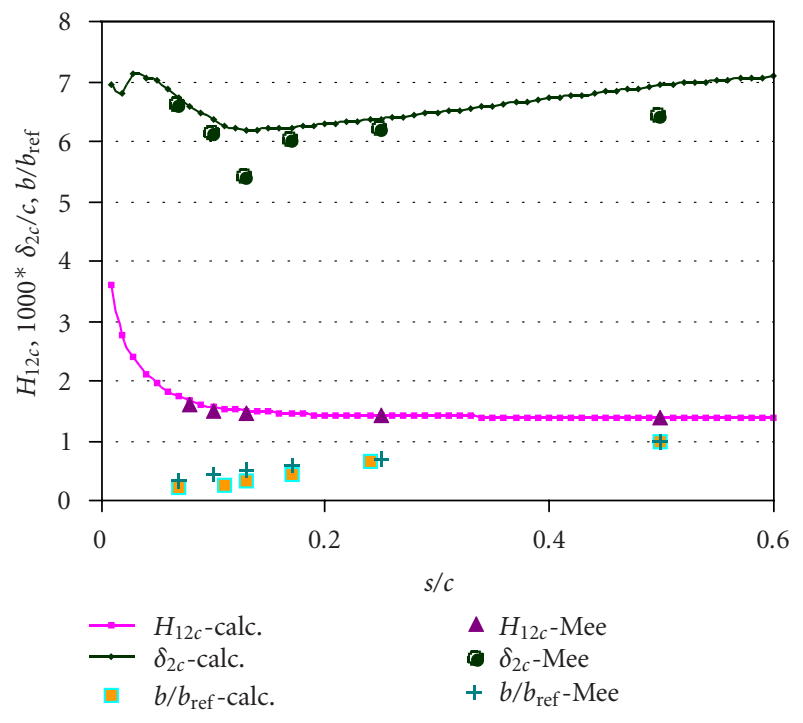

FIgURE 3: Comparison of prediction and test data: compressible form factor $H_{12 c}$, wake width $b / b_{\text {ref }}$, and momentum thickness $\delta_{2 c} / c$ for the cascade tested by Mee et al. [2].

The ratio of the compressible and incompressible form factors then becomes

$$
\frac{H_{12 c}}{H_{12}}=\frac{\left(F-k_{1} F_{2}\right) \cdot\left(\left(k_{3}+k_{1}\right) F-k_{1} k_{3} F_{2}\right)}{\left(k_{1} F-\left(k_{1} k_{3}+k_{1}^{2}\right) F_{2}+k_{3} k_{1}^{2} F_{3}\right) \cdot F}
$$

which is consistent with the experimental results in Kurz [8]. We will need this correlation later to calculate the velocity deficit at the wake centerline from the compressible form factor.

\section{BEHAVIOR OF THE FORM FACTOR}

The equations for the behaviour of the form factor are based on an approach by Spence [9] for incompressible wakes. We found, however, that Spence's [9] approach is only valid for the initial part of the wake development. Two zones can be distinguished in the development of the wake as evident from Figure 3.

In zone 1 , we find a rapid decrease of $H_{12 c}$. This behavior can be described with a modification of Spence's [9] equation, replacing the incompressible form factor with the compressible form factor $H_{12 c}$ :

$$
H_{12 c}=\frac{1}{1-(40 \cdot s / c+1)^{m} \cdot\left(1-1 / H_{T c}\right)} .
$$

For zone 2, we found a much slower decrease of $H_{12 c}$. It seems that zone 2 starts when the wake width exceeds a certain value compared to the pitch of the cascade. However, the experimental data is not sufficient to determine that ratio of $2 b /\left(t^{*} \sin \alpha_{1}\right)$ precisely enough (it has approximately the value of 0.2 ) to make it a criterion for switching from zone 1 to zone 2. Instead, the criterion was to use whichever the larger value for $H_{12 c}$ of the two equations was.

The relation for $H_{12 c}$ in zone 2 was determined to be

$$
\begin{gathered}
H_{12 c}=1.4593 \cdot f\left(M^{*}\right) \cdot\left(\frac{s}{c}+1\right)^{-0.1^{*}\left(1+m_{L}\right)}, \\
f\left(M^{*}\right)=\sqrt[5]{M^{*}}, \quad 0.8 \prec M^{*} \prec 1.0, \\
f\left(M^{*}\right)=\sqrt[3]{M^{*}}, \quad M^{*} \leq 0.8 .
\end{gathered}
$$

This data fit is validated for Laval numbers between 0.7 and 1.0. For flows with low Laval numbers, the wake typically does not leave the realm of zone 1 , that is, the equation for zone 1 is valid for the entire wake.

It is important to keep in mind that the form factor represents the velocity decay of the wake. We cannot therefore assume that $m$ is a constant. As found by Raj and Lakshminarayana [10], the decay of the velocity defect depends strongly on the flow field outside of the wake. This means that $m$ in (13) and (14) will depend on the behavior of the velocity at the wake edge.

We assume that the known behavior of $U$ can generally be described by $U \sim s^{m}$ with $m$ being constant for small segments of $s$. The relation between two adjacent points is then

$$
m=-0.5\left(1+m_{L}\right), \quad m_{L}=\frac{\log \left(U\left(s_{1}\right) / U\left(s_{2}\right)\right)}{\log \left(s_{1} / s_{2}\right)} .
$$

The aforesaid does not yet take into consideration the effect of a nonsymmetrical wake, as it typically will appear behind cascades due to the different boundary layers on suction and pressure side. Spence [9] has concluded that, given the definition of the initial form factor as used in (13), one can neglect the effect of nonsymmetrical wakes on the behavior of this form factor.

As mentioned before, one of the crucial points in this calculation is the form factor at the trailing edge. Because the base pressure plays an important role in the loss generation of a turbine blade, we have to take this into consideration for a revised definition of the form factor at the trailing edge. We assume the influence of the trailing edge as follows: The displacement thickness is equal to the trailing-edge thickness as before. The base pressure creates a loss, which is then transferred into a momentum thickness by

$$
\delta_{2 t e}=\frac{\delta_{t e} c_{p b}}{2}
$$

using Fiedler's [11] definition of the loss due to the base pressure.

This leads ultimately to a form factor at the trailing edge:

$$
H_{T}=\frac{\delta_{1 s s}+\delta_{1 p s}+\delta_{t e}}{\delta_{2 s s}+\delta_{2 p s}+\left(\delta_{t e} c_{p b} / 2\right)} .
$$

The contribution of the trailing-edge thickness to the denominator can almost be neglected, whereas especially for 
blades with thick trailing edges, the contribution of the trailing-edge thickness to the overall form factor is considerable.

Figure 3 shows the validity of the present approach by using data from Mee et al. [2].

\section{ASSUMPTION ABOUT THE POTENTIAL FLOW FIELD}

As stated previously, the inviscid flow field has a great impact on the development of the wake. Typically, the structure of the inviscid flow field is quite complex. In order to implement the inviscid flow field onto the present model, we use the following assumptions, which are backed by Schlieren visualizations, for example, by Nakayama [12].

(i) The flow field can be described by a constant velocity with superposed cosine waves.

(ii) The amplitudes of the cosine waves decline with $\exp \left(-k_{4} z\right)$, with $k_{4}=3.815$ from experimental data by Kurz [8].

(iii) The initial amplitude (at $z=0$ ) is half the difference between the highest Laval number on the suction side and the velocity at the trailing edge.

(iv) The maxima are located on a line perpendicular to the suction surface at the location of the highest velocity. They proceed at an angle $\gamma$ to the axial direction. This requirement defines also $k_{7}$.

(v) The minima are located on lines parallel to the maximum locations, but through the trailing edge.

(vi) If the velocity maximum appears at or close to the throat, we assume axial propagation.

As experimental data (Kurz $[4,5])$ shows, the static pressure field across the wake is imposed by the inviscid flow field. The above assumptions lead to a the following description of the inviscid flow field behind a cascade:

$$
\begin{aligned}
U(s, r)= & \bar{U}-\Delta U_{0} \\
& \cdot \exp \left(-k_{4}\left(s \sin \left(\alpha_{1}-\gamma_{1}\right)-r \cos \left(\alpha_{1}-\gamma_{1}\right)\right)\right) \\
& \cdot \cos \left(\frac{2 \pi\left(s \cos \left(\alpha_{1}-\gamma_{1}\right)+r \sin \left(\alpha_{1}-\gamma_{1}\right)+k_{8}\right)}{\left(t+k_{9} k_{7}\right) \cos \left(\gamma_{1}\right)}\right), \\
\frac{\Delta U_{0}}{\bar{U}}=\frac{\Delta M^{*}}{2 M^{*}}, & \quad k_{9}=-1 \wedge k_{8}=0, \\
& s<\frac{\left(t-k_{7}\right) \cdot \cos \left(\gamma_{1}\right)}{2 \cos \left(\alpha_{1}-\gamma_{1}\right)}, \quad k_{9}=1 \wedge k_{8}=k_{7} \cos \left(\gamma_{1}\right) .
\end{aligned}
$$

\section{BEHAVIOR OF MOMENTUM AND DISPLACEMENT THICKNESS}

To determine the development of $\delta_{1 c}$ and $\delta_{2 c}$, we now apply the boundary layer momentum equation, modified for wakes
TABLE 1: Configurations for sample calculations.

\begin{tabular}{lccc}
\hline Source & Kurz [5] & Kurz [5] & Mee et al. [2] \\
\hline$t / c$ & 0.877 & 0.822 & 0.84 \\
$M_{1}^{*}$ & 0.96 & 0.71 & 0.93 \\
$\alpha_{1}$ & 27.0 & 25.5 & 22.0 \\
$\delta_{t e} / c$ & 0.04 & 0.04 & 0.02 \\
$K_{7} / t$ & -0.69 & +0.41 & +0.65 \\
$\Delta M^{*} / M^{*}$ & 0.41 & 0.12 & 0.10 \\
$\gamma$ & 23.0 & 0.00 & 19.0 \\
$H_{T}$ & 22.0 & 17.0 & 6.9 \\
$\operatorname{Re} / 10^{5}$ & 6.7 & 4.5 & 10.0 \\
\hline
\end{tabular}

insofar as the shear stress at the wake centerline is zero:

$$
\frac{d \delta_{2 c}}{d s}+\delta_{2 c} \cdot\left(\frac{H_{12 c}+2}{U} \cdot \frac{d U}{d s}+\frac{1}{\rho_{s}} \frac{d \rho_{s}}{d s}\right)=0 .
$$

The values for $U, d U / d s$, and $\rho$ are known from the assumption on the potential flow. $H_{12 c}$ is calculated using (13) and (14).

Because of the nature of cascade flows, we must correct the average free-stream velocity $U$ by the flow into or out of the wake region. This is done by

$$
\bar{U}=\frac{\left(t \sin \alpha_{1}-\delta_{1 c}\right)_{\text {init. }}\left(\rho_{s} U\right)_{\text {init. }},}{\left(t \sin \alpha_{1}-\delta_{1 c}\right) \cdot \rho_{s}},
$$

where the initial values are taken at the trailing edge.

Figure 3 shows the validity of the described assumptions. It shows especially that $\delta_{2 c}$ is highly influenced by the freestream velocity. In Figure 3, where the calculated results are compared with Mee's [2] data, it can be seen that the model is able to capture the decreasing and increasing of $\delta_{2 c}$, which is obviously caused by the flow conditions outside of the wake.

\section{TEST CALCULATIONS AND COMPARISON WITH TEST RESULTS}

The set of equations derived above allows to calculate typical shapes of wakes as inputs into other calculations without having to perform a complete Navier-Stokes calculation.

In order to prove its usefulness, several test calculations were performed to compare them with data of several configurations (Table 1).

All the test calculations are for transonic turbine cascade, typically, with high turning. The cascade tested by Kurz [3, $4,5]$ is a nozzle profile from a small gas turbine with a very thick trailing edge and an exit flow angle of about $26^{\circ}$. Mee et al. [2] tested a turbine profile with a fairly thick trailing edge and a flow angle of $22^{\circ}$. Table 1 lists pertinent data for the configurations.

Having calculated $\delta_{2 c}$ and $H_{12 c}$ for a certain location behind the blade, it is possible to calculate the wake width and velocity defect. From $H_{12 c}$, we calculate $H_{12}$ using (12) which leads to $u_{0} / U=k_{1}$. We now can calculate $k_{3}$, and reiterate the calculation of $H_{12}$, now using (10). $\delta_{2 c}$ leads to $\delta_{1 c}$, which 


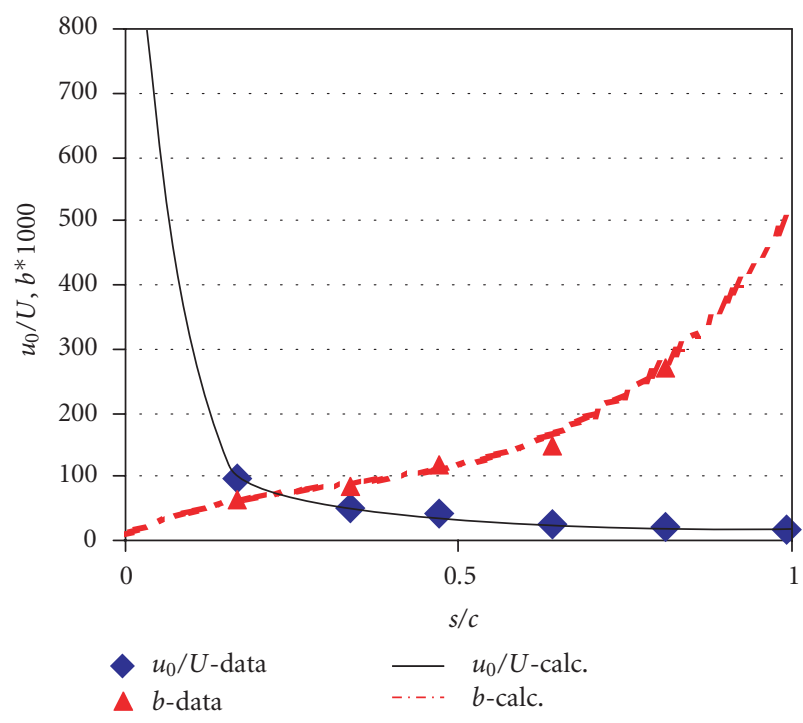

(a)

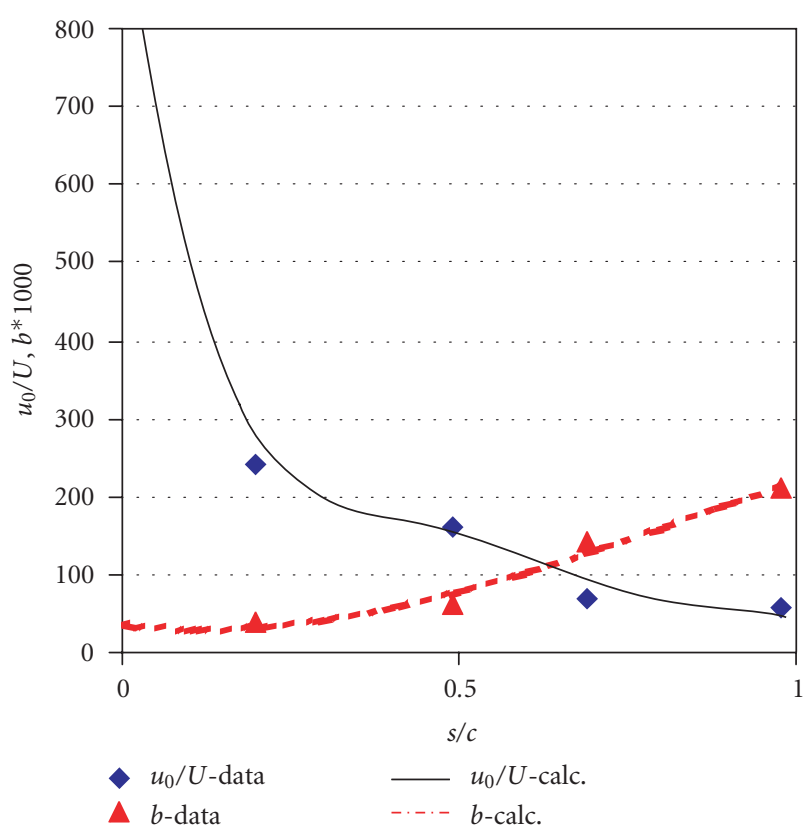

(b)

Figure 4: Measured and calculated velocity defects and wake width for cascades tested by Kurz [5]: (a) $t / c=0.877$ and $M^{*}=0.96$, and (b) $t / c=0.822$ and $M^{*}=0.71$.

together allow to determine the values of $k_{2}$. Figures 3 and 4 show comparisons between calculated and measured downstream development of the wake for the data reported by Mee [2] and Kurz [5]. With this information, we can compute the loss distribution either parallel to the exit plane or perpendicular to $s$. Figure 4 shows a comparison between calculated and measured velocity defects $u_{0} / U$ and wake width $b=2 k_{2}$, respectively. We can see in Figure $4 \mathrm{~b}$ that for $M^{*}=0.71$ the switch from zone 1 to zone 2 does not appear until $s / c=0.65$.

\section{CONCLUSIONS AND PRACTICAL IMPORTANCE}

The results of this paper show that no simple solution for the wake characteristics exists for transonic turbine blades. An important reason for this is the fact that the inviscid flow field imposes complex boundary conditions onto the wake. The presented model is able to simulate wakes of turbine blades very accurately because a simple but realistic model for the inviscid flow field behind the cascade was created.

The results show that the dominating factors for the formation of the wake are the inviscid velocity field behind the cascade and the form factor at the trailing edge $H_{T c}$, which is mostly determined by the trailing edge thickness. There is also a very distinct influence of the average free-stream Laval number. Especially, the wake width, which is influenced by the local momentum thickness in addition to the form factor, shows a distinct dependency on the inviscid flow field even far downstream of the trailing edge. We did not find an indication for an influence of the Reynolds number. Also, the free-stream turbulence seemed to play a negligible role. The information compiled in this paper allows to select realistic parameters for the description of wakes. Therefore, realistic input parameters in numerical flow calculations can be easily selected. Results from CFD calculation can be verified against the described, very simple, and universal correlations.

\section{ACKNOWLEDGMENTS}

The author wishes to thank Prof. Dr.-Ing. Fiedler and Aziz Fozi for their helpful discussions. All the measurements were conducted at the "Laboratorium für Strömungsmaschinen" at the University of the German Armed Forces in Hamburg.

\section{REFERENCES}

[1] O. Sieber, "Quantitative Dichtefeldmessung mit RayleighStreulicht am Laufschaufelprofil VKI-1 und Vergleich mit einer numerischen Simulation," VDI Fortschrittberichte Nr. 301, VDI-Verlag, Duesseldorf, 1996.

[2] D. J. Mee, N. C. Baines, M. L. G. Oldfield, and T. E. Dickens, "An Examination of the Contributions to Loss on Transonic Turbine Blade in Cascade," ASME Paper 90-GT-264, 1990.

[3] R. Kurz, "The gas flow behind a cascade with nonuniform pitch," ASME FED, vol. 101, pp. 73-81, 1990.

[4] R. Kurz, "Transonic flow through turbine cascades with 3 different pitch to chord ratios," in Proc. 10th International Symposium Air Breathing Engines (ISABE '91), pp. 659-668, Nottingham, UK, September 1991.

[5] R. Kurz, Experimentelle und theoretische Untersuchungen an gleichfoermig und ungleichfoermig geteilten Turbinengittern, Ph.D. dissertation, University of the Federal Armed Forces, Hamburg, Germany, 1991.

[6] H. Schlichting, Ed., Boundary Layer Theory, McGraw Hill, New York, NY, USA, 1987.

[7] H Reichardt, "Gesetzmaessigkeiten der freien Turbulenz," VDI Forschungsheft 414, 1942.

[8] R. Kurz, "Effects of nonuniform pitch on the flow through an annular turbine nozzle," Int. Journal of Rotating Machinery, vol. 2, no. 1, pp. 59-65, 1995.

[9] D. A. Spence, "Growth of the Turbulent Wake Close Behind an Airfoil at Incidence," ARC CP 125, 1952. 
[10] R. Raj and B. Lakshminarayna, "Characteristics of the wake behind a cascade of airfoils,” J. Fluid Mech., vol. 61, pp. 707730, 1973.

[11] K. Fiedler, Ueber die Verluste in ebenen Turbinenschaufelgittern, Habilitation dissertation, University Hannover, Hannover, Germany, 1966.

[12] Y. Nakayama, Ed., Visualized Flow, Pergamon Press, Oxford, England, 1988. 

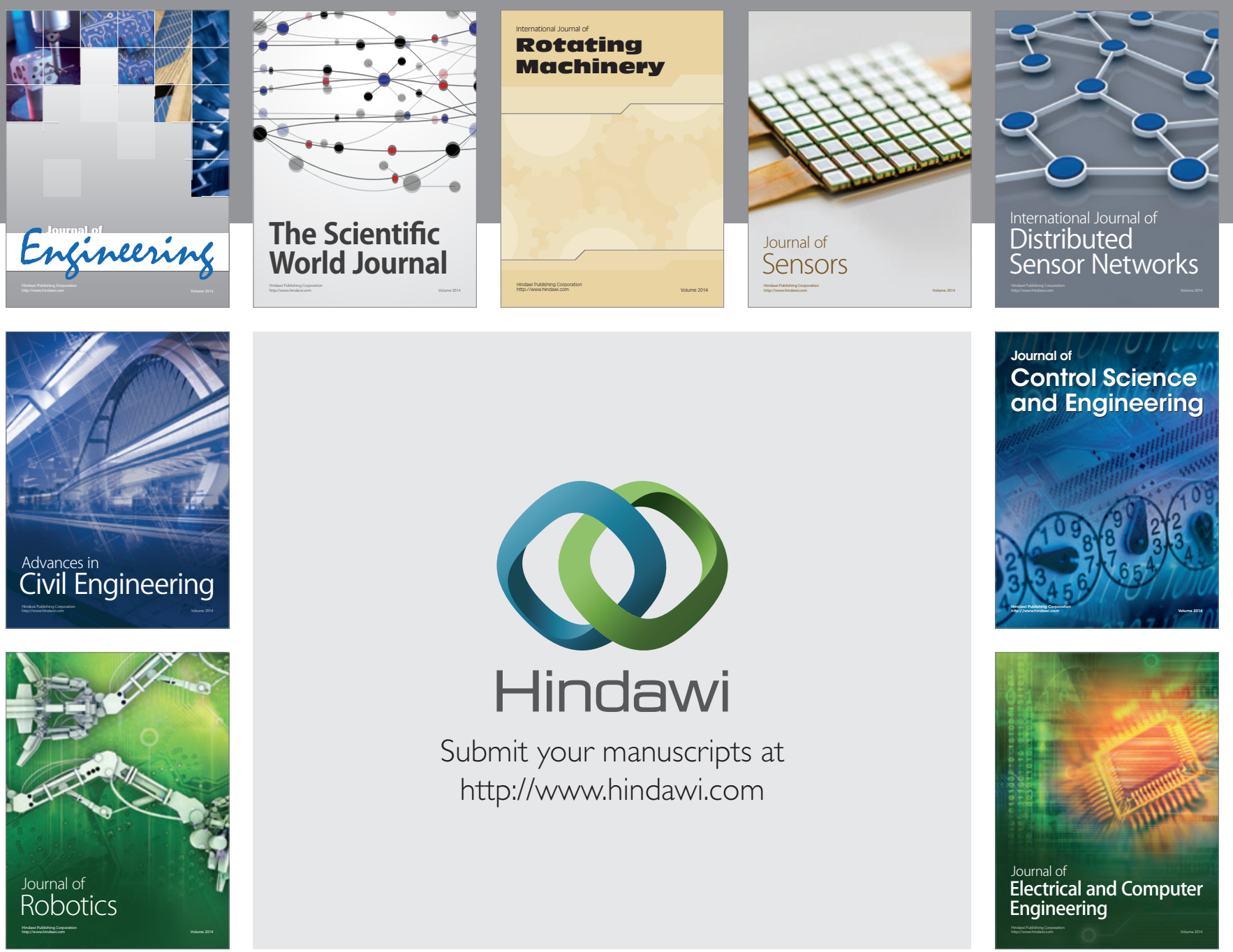

Submit your manuscripts at

http://www.hindawi.com
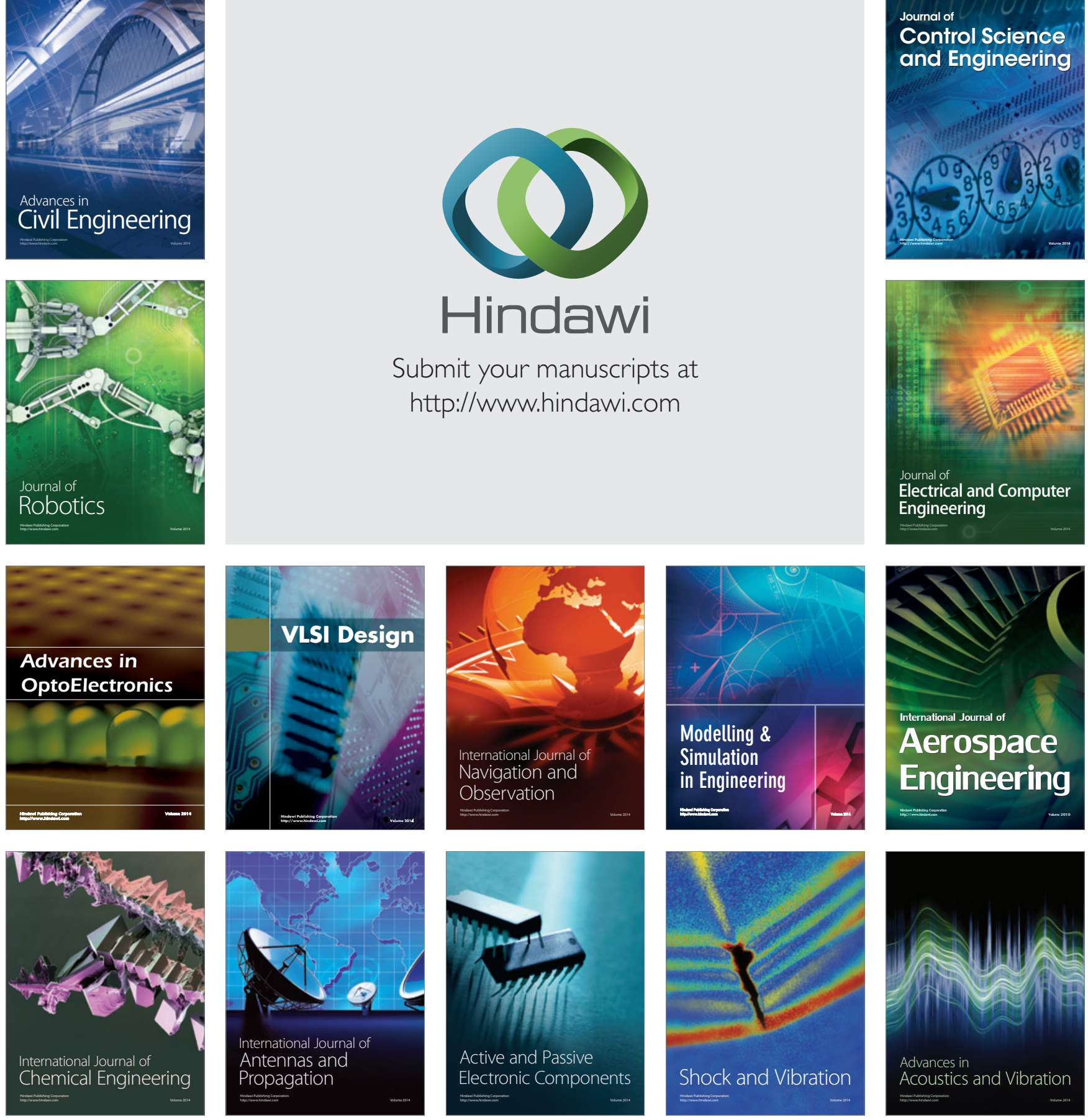